3. Medication safety in neonatal care involves education and training of the staff; debriefs and shared learning from errors, and timely review of the practices.

\section{IS IGNORANCE REALLY BLISS? EFFECT OF PARENTAL AWARENESS ON NEONATAL MORTALITY IN CENTRAL INDIA}

Amruta Phatak, Abhimanyu Niswade, Dipty Jain, Khushboo Khubchandani. India

\subsection{6/bmjpo-2021-RCPCH.219}

Background Only with education can we create generalized awareness regarding better neonatal care at the societal level. It is well known that the people in many rural and tribal areas across the developing world tend to harbor grave misconceptions regarding pregnancy and neonatal care and lack the knowledge of danger signs. However, the level of parental awareness and its effect on neonatal mortality in the intensive care units in urban areas is often overlooked.

Objectives To assess the effect of parental awareness on the survival outcome of early preterm neonates in a tertiary care center providing referral services.

Methods After taking the approval of the institutional ethics committee, a cross sectional quantitative social research study was conducted. 140 neonates of gestational age ranging between 28-31 weeks and birth weight ranging from $1000 \mathrm{~g}$ to $2499 \mathrm{~g}$; of both sexes born in a tertiary care institute of central India and admitted to its neonatal intensive care unit (NICU) within 1 hour of birth were enrolled in the study. Babies of gestational age $<28$ weeks and $>31$ completed weeks, birth weight $<1000 \mathrm{~g}$, having lethal congenital malformations, delivery room deaths and those admitted after 1 $\mathrm{hr}$ of birth were excluded. The outcome measure was in the form of hospital death or discharge. The awareness of parents of the enrolled neonates, regarding neonatal care and danger signs, was assessed using a single response (yes/no type) 5 questions questionnaire, prepared in local languages. For parents referred from tribal areas, assistance was taken from translators available among relatives or hospital staff. Their responses were correlated with the outcome. The 'Yes' answers to $80 \%$ of the questions were considered a positive response, which was taken as an indicator of good awareness. Chisquare test and $2 \times 2$ table were used and the p-value was calculated; $\mathrm{p}<0.05$ was considered statistically significant. The questions asked were regarding the parents being aware of their baby's low birth weight or preterm status, its associated complications, benefits of breastfeeding, identification of each of the danger signs, and whether their response to those signs will be to consult a doctor immediately or to try homebased treatment first.

Results Male: female ratio was 0.92:1. The mean Gestational age was $30.27 \pm 0.89$ weeks, the mean birth weight being $1599.75 \pm 282.35$ grams. The total mortality in the cohort was $47.1 \%$ (66/140). For the mortality group (66), only $42.31 \%$ of parents gave a positive response. The survived group (74) was associated with a $58.30 \%$ positive response. Thus, the higher the positive response rate greater was the survival $(\mathrm{p}<0.007)$.

Conclusions Increased sensitization of parents regarding neonatal danger signs and care practices has a positive impact on neonatal survival, highlighting that parental education is the need of the hour. Improved parental education from the grass-root level in rural areas up to the tertiary care centers in urban areas, regarding safe neonatal health practices, will help in reducing neonatal mortality rate and in achieving target 3 of Sustainable Development Goals.

\section{FIVE YEAR EVALUATION OF THE NEWBORN SCREENING PROGRAMME IN DUBAI, UNITED ARAB EMIRATES: A CROSS SECTIONAL STUDY}

Mariam Elsaban, Donia Ahmad, Nagham JA Toba, Thannon Alsaeed, Zahreddin Guma Abusalah. United Arab Emirates

\subsection{6/bmjpo-2021-RCPCH.220}

Background The Newborn Screening Programme (NBS) screens for a group of congenital and genetic conditions including the inborn errors of metabolism. The population of the United Arab Emirates' presents uncharted ground for exploration of these disorders. High rates of consanguinity contribute to the higher prevalence of these rare diseases. Early diagnosis is key to lead to better prognosis.

Objectives To investigate the frequency of various neonatal disorders detected by a comprehensive newborn screening programme conducted in a tertiary care hospital over 5 years.

Methods A total of 14733 neonates born in our hospital between October 2012 and October 2017 were included in the study. Neonates underwent a heel prick test at 48 hours of birth which was sent for screening of various disorders. The results of these tests were collected as well as demographic variables including, gender, birth weight, gestational age and birth year. Statistical analysis was carried out through Statistical Package for the Social Sciences SPSS v.24. Qualitative variables were tabulated as frequencies and percentages and the continuous variables as means \pm Standard deviation.

Results Of the 14,733 neonates 7049 (47.85\%) were females. Average gestational weeks and weight were $37.9( \pm 2)$ weeks and $3127( \pm 561)$ grams respectively. $14(0.1 \%)$ tested positive for inborn errors of metabolism. Of whom $2(0.01 \%)$ had fatty acid disorders, $11(0.07 \%)$ had amino acid disorders, and 1 had cystic fibrosis. Of the amino acid disorders, 2 neonates had Phenylketonuria. Three (0.02\%) newborns tested positive for congenital adrenal hyperplasia and 8 $(0.05 \%)$ for congenital hypothyroidism. 161 (1.1\%) newborns tested positive for glucose-6-phosphate dehydrogenase deficiency and another $172(1.2 \%)$ were carriers. Breakdown of genetic analysis for glucose-6-phosphate dehydrogenase deficiency revealed the most common mutation being C563T Mediterranean mutation occurring in 115 (71\%) of neonates. Furthermore, 296 (2\%) newborns were found to have a variety of hemoglobinopathy spectrum with only another 13 (0.09\%) newborns being positive for a hemoglobinopathy disorder.

Conclusions Glucose-6-phosphate dehydrogenase deficiency G6PD was the most commonly detected condition. However, this rate is lower than these reported in previous studies. Hemoglobinopathy carriers were the highest detected abnormality. A higher frequency of inborn errors of metabolism, hemoglobinopathies, congenital hypothyroidism, and congenital adrenal hyperplasia than worldwide figures was noted. Several factors such as higher regional consanguinity rates and a multi-ethnic population were identified as potential explanations. 
The study provides valuable information on a number rare disorders in the UAE. Early diagnosis and treatment leading to better prognosis is an important gain. Moreover, these results equip healthcare leaders with new information on the burden of these conditions to ensure better planning and provision of health services.

\section{CARER AND PATIENT PERSPECTIVE ON CLINICAL AND RESEARCH NEEDS TO IMPROVE CARE FOR FOOD PROTEIN INDUCED ENTEROCOLITIS}

Shubhasree Mukherjee, Rebecca Knibb, Ekaterina Khaleva, Marta Vazquez-Ortiz. UK

\subsection{6/bmjpo-2021-RCPCH.221}

Background Food Protein induced enterocolitis (FPIES) is a non-Immunoglobulin E(Ig-E) mediated gastrointestinal food allergy affecting mainly children. It has a significant impact on quality of life and despite being a potentially life-threatening allergy, awareness is poor and the diagnosis is often missed or delayed, as 'it does not look like an allergy' and IgE-based allergy tests are usually negative. There is very limited data on parent/patient perspectives of the unmet clinical or research needs in FPIES that can affect clinical outcomes.

Objectives To identify unmet research and clinical needs, as perceived by patients with FPIES and carers of children with FPIES, to deliver value-based patient care.

Methods An online survey was disseminated in English through the USA-based patient/parent association 'FPIES Foundation', FPIES UK, Food Allergy and Anaphylaxis Australia, Food Allergy Canada and Allergy Italia, between May 2020 and July 2020. Participants included adults with self-reported FPIES or a carer of a child (0-18 years) with FPIES. The survey collected demographic data of patients, incidence of acute and chronic FPIES, parent and patient perspectives of unmet research needs and psychological effect of FPIES on families, including ranking a number of potential needs from 1 (not essential) to 9 (extremely essential). The survey contained closed and open-ended questions; the latter were analysed using thematic analysis.

Results A total of 285 respondents completed the survey, of which $255(89.5 \%)$ were parent/carer of a child with FPIES (0-18 years), 21 (7.4\%) were adults with FPIES and 9 (3.2\%) were adult with FPIES themselves and a parent/care-giver of a child with FPIES. Female participants comprised 93\% (265/ $285)$ of the total and $49.5 \%(141 / 285)$ belonging to the age group of $35-44$ years.

Majority $(76 \%, 182 / 239)$ of children had FPIES to multiple foods, the commonest culprit food being grains other than gluten i.e. rice/oats $(62 \%, 148 / 239)$. 48.75\% (117/240) of children suffered from chronic with acute FPIES followed closely by children with acute FPIES only (46.25\%, 111/240).

The top research priorities identified by participants (in decreasing order of importance) were 1.) Creating awareness about FPIES amongst health care professionals(HCP), in particular emergency doctors and nurses; 2.) Education of medical students on FPIES, 3.) The development of a prognostic and predictive test for FPIES, 4) An established care plan for continuity of care following visit to emergency and 5) A diagnostic test to diagnose FPIES acutely against common differentials.

The major themes identified in the data were 1.) Creating awareness about FPIES amongst HCP and in community, 2.)
FPIES is psychologically stressful 3.) FPIES leads to social restriction due to fear of accidental ingestion leading to severe symptoms 4) FPIES is financially stressful and should be covered by health insurance and 5) Lack of stringent foodlabelling.

Conclusions Increasing awareness amongst healthcare professionals to avoid misdiagnosis/delay and lack of a prognostic/diagnostic test for FPIES were the key research needs. Further research and clinical improvement strategies should be focused on these areas.

\section{CHILDHOOD OBESITY: A SURVEY OF KNOWLEDGE AND PRACTICES OF PEDIATRICIANS AND PEDIATRIC RESIDENTS}

Li Ming Ong, Kai Guo Benny Loo, Jean Yin Oh, Elaine Chew. Singapore

\subsection{6/bmjpo-2021-RCPCH.222}

Background Childhood obesity is one of the most common and serious public health issues in the twenty-first century. Overweight and obese children are more likely to stay obese into adulthood and develop cardiovascular diseases at a younger age. Now, pediatricians have to manage these conditions that were once regarded as adult diseases.

Objectives The main aims are: 1. Identify knowledge gaps in medical, dietary, exercise and psychological aspects of childhood obesity; 2. Highlight the challenges in treating obese patients.

Methods This is a single-center, cross sectional quantitative survey of pediatricians and pediatric residents working in the largest tertiary children hospital in Singapore (KK Women's and Children's Hospital). Baseline demographic data as well as participants' knowledge in medical, dietary, physical activity and psychological aspects of childhood obesity were collected using a 32-question survey. A group of medical and allied health professionals designed and reviewed the survey. The correct answers for BMI cut-offs for obesity and overweight, healthy balanced diet in proportion of plate, recommended sugar intake, physical activity for children were defined according to national and international guidelines. Junior doctors comprised of house officers, medical officers and junior residents. Senior doctors were made up of senior residents and pediatricians.

Results A total of 123 doctors were surveyed (46\% were juniors). Both juniors and seniors tend to encounter overweight and obese patients on weekly basis. The most common challenge cited during patient consultations was patient motivation-related. Expectedly, the seniors perceived themselves to be more comfortable and knowledgeable compared to juniors. Other common challenges were lack of time and other medical conditions taking precedence over weight concerns. Physical activity recommendation was the weakest area, followed by dietary advice. There was no significant differences in medical, dietary, physical activity and psychological knowledge associated with obesity between junior and senior doctors.

Conclusions There were similar knowledge gaps in all domains of childhood obesity between junior and senior doctors. Physical activity recommendation is the weakest area. Patient motivation is the commonest challenge faced. Hence, future education programs should target all doctors and focus on motivational interviewing. 